\title{
HEart and BRain interfaces in Acute ischemic Stroke (HEBRAS) - rationale and design of a prospective oberservational cohort study
}

\author{
Karl Georg Haeusler ${ }^{1,2^{*}}$, Ulrike Grittner ${ }^{1,7}$, Jochen B. Fiebach ${ }^{1}$, Matthias Endres ${ }^{1,2,3,4,5,6}$, Thomas Krause ${ }^{1,2}$
} and Christian H. Nolte $e^{1,2}$

\begin{abstract}
Background: An effective diagnostic work-up in hospitalized patients with acute ischemic stroke is vital to optimize secondary stroke prevention. The HEart and BRain interfaces in Acute ischemic Stroke (HEBRAS) study aims to assess whether an enhanced MRI set-up and a prolonged Holter-ECG monitoring yields a higher rate of pathologic findings as compared to diagnostic procedures recommended by guidelines (including stroke unit monitoring for at least $24 \mathrm{~h}$, echocardiography and ultrasound of brain-supplying arteries).

Methods/Design: Prospective observational single-center study in 475 patients with acute ischemic stroke and without known atrial fibrillation. Patients will receive routine diagnostic care in hospital as wells as brain MRl, cardiac MRI, MR angiography of the brain-supplying arteries and Holter-monitoring for up to 10 days. Study patients will be followed up for cardiovascular outcomes at 3 and 12 months after enrolment.

Discussion: By comparing the results of routine diagnostic care to the study-specific MRI/ECG approach, the primary outcome of HEBRAS is the proportion of stroke patients with pathologic diagnostic findings. Predefined secondary outcomes are the association of stroke localization, autonomic dysbalance and cardiac dysfunction as well as the effect of impaired heart-rate-variability on long-term clinical outcome.

The investigator-initiated HEBRAS study will assess whether an enhanced MRI approach and a prolonged ECG monitoring yield a higher rate of pathological findings than current standard diagnostic care to determine stroke etiology. These findings might influence current diagnostic recommendations after acute ischemic stroke. Moreover, HEBRAS will determine the extent and clinical impact of stroke-induced cardiac damage.
\end{abstract}

Trial registration: Clinicaltrials.gov NCT02142413.

Keywords: Atrial fibrillation, Cardiac MRI, ECG monitoring, Ischemic stroke, Heart rate variability, Autonomic dysfunction

\section{Background}

Variations and delays in the diagnostic procedures during hospitalization after acute ischemic stroke are common [1-3]. At the same time, stroke etiology remains cryptogenic in about $20-25 \%$ of stroke unit patients [4].

Recent studies have shown that magnetic resonance imaging (MRI) and MR angiography are able to detect

\footnotetext{
* Correspondence: georg.haeusler@charite.de

'Center for Stroke Research Berlin, Charité - Universitätsmedizin Berlin, Berlin, Germany

${ }^{2}$ Department of Neurology, Campus Benjamin Franklin, Charité

Universitätsmedizin Berlin, Hindenburgdamm 30, 12200 Berlin, Germany

Full list of author information is available at the end of the article
}

cardiac [5, 6], aortic [7] as well as carotid sources of embolism with comparable sensitivity as compared to diagnostic ultrasound [8]. Moreover, prolonged ECG monitoring can significantly increase the detection rate of atrial fibrillation (AF) $[9,10]$. The detection of AF implies a four- to fivefold increased risk of stroke [11]. These developments may allow for a more effective diagnostic work-up in patients with acute ischemic stroke as compared to standard diagnostic procedures, namely ultrasound of the brain-supplying arteries, echocardiography and stroke unit monitoring. However, there is no common standard of stroke unit monitoring [12] and guidelines [13] do not strictly recommend prolonged 
ECG monitoring [14]. In addition, availability of echocardiography is often limited $[1,15]$, and a substantial number of stroke patients are not willing or able to undergo semi-invasive transesophageal echocardiography, while transthoracic echocardiography is not sufficient for assessing aortic plaque and left atrial thrombi.

The prospective observational HEart and BRain interfaces in Acute ischemic Stroke (HEBRAS) study therefore aims to assess the detection rate of pathologic findings relevant to stroke etiology as obtained by an enhanced MRI set-up (including brain MRI, cardiac MRI, MR angiography of the brain-supplying arteries) and a prolonged, non-invasive, commonly available Holter monitoring (of up to 10 days duration) in comparison to findings obtained by routine diagnostic procedures after acute stroke. In addition, the underlying pathophysiological mechanisms and the prognostic impact of stroke-induced cardiac damage are still poorly understood $[16,17]$. For that reason, the investigator-initiated HEBRAS study attempts to shed more light on the relationship between stroke localization (e.g., insular involvement), observed cardiac damage (as indicated by troponin elevation) and the activation of the autonomic nervous system (as indicated by impairment of heart rate variability and elevated urinary norepinephrine levels). Moreover, stroke-induced autonomic dysfunction and observed cardiac damage will be analyzed with respect to functional outcome, mortality, recurrent stroke and myocardial infarction during a one year follow-up.

\section{Methods}

\section{Study design}

The Center for Stroke Research Berlin (CSB) is the sponsor of the investigator initiated HEBRAS study, which is supported by a grant from the German Federal Ministry of Education. The study was approved by the scientific advisory board of the CSB in September 2013, and by the Ethics Committee of the Charité - Universitätsmedizin Berlin, Germany (EA2/033/14) in March 2014. All study procedures are carried out in accordance with the principles of Good Clinical Practice and the Declaration of Helsinki. All study patients have to fulfill the study entry criteria listed in Table 1 including written informed consent.

Study patients will receive routine diagnostic procedures in hospital (defined as stroke unit monitoring (according to clinical needs), echocardiography, ultrasound of the brain-supplying arteries, and 24-hour Holter-ECG (if indicated according to the treating physician)). Moreover, study patients will undergo an enhanced MRI work-up (comprising brain as well as cardiac MRI, MR angiography of the aortic arc and the supra-aortic, brain-supplying arteries) combined with Holter-ECG of up to 10 days (including a maximum of 5 days after
Table 1 Inclusion and exclusion criteria of the HEBRAS study

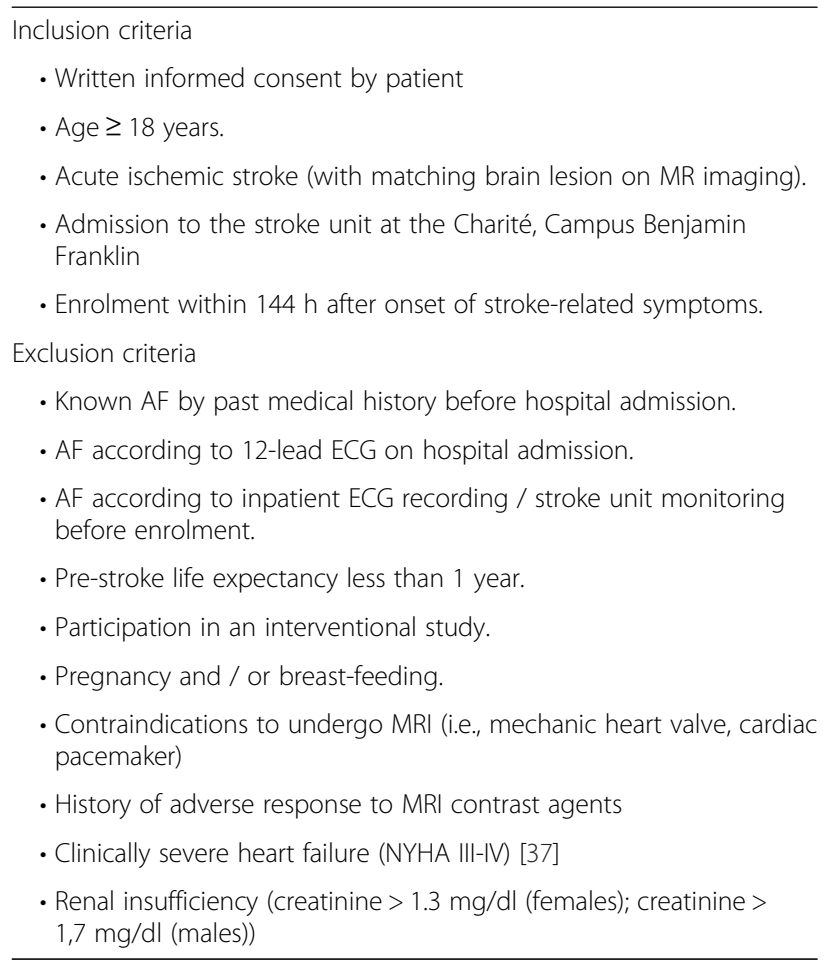

hospital discharge). For the primary outcome, the results of routine diagnostic care will be compared to studyrelated diagnostics. Results of the ECG core lab analysis will be provided to study patients and treating physicians. Study patients will be followed up for cardiovascular outcomes e.g., recurrent stroke, major vascular events, death and degree of dependency at 3 and 12 months after enrollment.

\section{Stroke etiology}

Etiology will be determined according to TOAST [18] and ESUS criteria [4]. The following diagnostic findings will be regarded as pathologic and relevant to stroke etiology: atrial fibrillation of any type, sick sinus syndrome, bacterial or nonbacterial thrombotic endocarditis, ipsilateral carotid or intracranial stenosis of $\geq 50 \%$ according to NASCET [19], left atrial or left ventricular thrombus, atrial myxoma, acute myocardial infarction resulting in wall motion abnormalities, akinetic left ventricular segment, dilated cardiomyopathy, systolic dysfunction with reduced left ventricular ejection fraction $(<45 \%)$, aortic plaque of $\geq 4 \mathrm{~mm}$.

\section{Magnetic resonance imaging}

In HEBRAS, enhanced diagnostic MRI work-up is defined as brain MRI, cardiac MRI and MR-angiography of the aortic arc and the brain-supplying arteries. All MR examinations are performed on a 3 Tesla MR scanner (TIM TRIO, Siemens, Erlangen, Germany). Cerebral 
MRI mainly consists of a T2*-sensitive sequence (TR $620 \mathrm{~ms}$, TE $20 \mathrm{~ms}$, flip angle $20^{\circ}$, FOV $220 \mathrm{~mm}$, slice thickness $5 \mathrm{~mm}$ ) to exclude intracerebral hemorrhage, a diffusion weighted (DWI) sequence (TR $7600 \mathrm{~ms}$, TE $93 \mathrm{~ms}$, FOV $230 \mathrm{~mm}$, slice thickness $2.5 \mathrm{~mm}$ ) for detection of ischemic tissue, and a 3D time-of-flight (TOF) angiographic sequence (TR $22 \mathrm{~ms}$, TE $3.86 \mathrm{~ms}$, flip angle $18^{\circ}$, FOV $200 \mathrm{~mm}$, slice thickness $0.65 \mathrm{~mm}$ ) for imaging of intracranial vessels. For cardiac imaging, initially a dark-blood-prepared HASTE (half-Fourier acquisition single-shot turbo spin-echo) sequence (TR $750 \mathrm{~ms}$, TE $49 \mathrm{~ms}$, Flip angle $160^{\circ}$, Slice thickness $5 \mathrm{~mm}$ ) is acquired, followed by 2 chamber as well as 4 chamber view acquisitions using a cine SSFP sequence (TR $40.56 \mathrm{~ms}$, TE $1.48 \mathrm{~ms}$, flip angle $50^{\circ}$, slice thickness $5 \mathrm{~mm}$ ) with prior scouting for individual frequency adjustment. Postcontrast imaging is performed after intravenous injection of $0.1 \mathrm{mmol} / \mathrm{kg}$ body weight Gadobutrol (Gadovist ${ }^{\circ}$, Bayer Schering Pharma, Berlin, Germany) with postcontrast cine imaging parameters held constant. For angiographic imaging of the aortic arch as well as extracranial cerebral arteries, a three-dimensional TWIST (Time-resolved angiography With Interleaved Stochastic Trajectories) sequence was used (TR $2.36 \mathrm{~ms}$, TE $0.99 \mathrm{~ms}$, Flip angle $20^{\circ}$, Slice thickness $1.40 \mathrm{~mm}$ ). Finally, myocardial late enhancement is characterised employing a turbo FLASH (Fast Low Angle SHot) sequence (TR $750.00 \mathrm{~ms}$, TE $1.97 \mathrm{~ms}$, Flip angle $20^{\circ}$, Slice thickness $8 \mathrm{~mm})$.

The following stratification of lesion localization is planned: left / right anterior cerebral artery, left / right middle cerebral artery, left / right posterior cerebral artery, left / right anterior choroideal artery, left / right insular cortex, left / right basal ganglia, left / right thalamus, left / right cerebellum, left / right brainstem, left / right medulla oblongata.

To bypass restrictive a priori assumptions on a specific relationship between stroke lesion and laboratory changes, an additional voxel-by-voxel analysis of lesion location and troponin-elevation will be conducted employing statistical principles developed for functional brain imaging $[20,21]$.

\section{Study ECG \& core lab analysis}

Additional ECG recording will be performed by using the portable CardioMem 4000 (GETEMED AG, Teltow, Germany). Study ECG will be started as soon as possible after enrolment and will be continued for a maximum of 10 days, i.e., two consecutive ECG measurements of five days. When patients are discharged before the first five days are completed, ECG recorder will be changed at discharge, resulting in an overall duration of less than ten days in Holter-ECG.
Study ECG data will be transmitted to the cardiac core lab at Department of Cardiology and Pneumology, Georg-August-University Göttingen, Germany for analysis. Raters analysing ECG-data will be blinded for clinical data including infarct localization. Core lab analysis will be provided to the respective study patient and the patient's treating physician (if indicated by clinical assumption).

The following definitions will be used throughout the study: Atrial fibrillation: Absolute arrhythmia lasting $\geq 30 \mathrm{~s}$ as defined in the current ESC guideline [22]. Short atrial tachycardia: Atrial tachycardia lasting $<30 \mathrm{~s}$ and consisting of $\geq 6$ conducted premature activations. Excessive supraventricular ectopic activity: More than three premature atrial complexes per hour [23]. Atrial flutter will be reported separately, and the study specifies to initiate anticoagulation for atrial flutter in the same way as for AF. The analysis will include: heart rhythm; PQ intervall $[\mathrm{s}]$, minimal [bpm], maximal [bpm] and mean heart rate (HR) [bpm], and measures of Heart rate variability (HRV) such as the standard deviation of beat-to-beat (NN) intervals (SD-NN), Root Mean Square of Successive Differences (rMSSD), HRV-Triangular Index (HRV$\mathrm{TI}$ ), low and high frequency (LF and HF, respectively) band and LF/HF Index [24].

\section{Baseline visit in hospital}

Baseline assessment of all study patients will include: a detailed analysis of patient demographics (also including time of stroke onset, hospital admission as well as admittance to the ward), clinical characteristics (including the National Institutes of Health Scale Score [25] and the modified Rankin Scale score [26], vital signs, medical history and concomitant diseases, extend and results of inpatient ECG recording, laboratory results (all data of clinical routine assessment plus high-sensitivity (hs) troponin and NT-proBNP), results of imaging (head CT/ MRI, carotid ultrasound of brain-supplying arteries, echocardiography) and TOAST classification at hospital discharge (before evaluation of the additional ECG monitoring), in hospital treatment (i.e., thrombolysis, ventilation etc.) as well as in hospital complications (such as pneumonia, recurrent stroke or myocardial infarction).

\section{Study follow-up}

Follow-up will be done 3 months and one year after the index stroke by a telephone interview conducted by trained personal of the Center for Stroke Research Berlin, Germany. The following data will be assessed: medication, recurrent ischemic stroke and other major vascular events, major bleeds, and the mRS score. Additionally, (all-cause) death and date of death will be registered using information from registration offices. 


\section{Study outcomes}

The primary outcome of the HEBRAS study is the rate of pathologic findings relevant to stroke etiology in patients with acute ischemic stroke obtained by enhanced diagnostic MRI work-up combined with prolonged Holter-monitoring in comparison to findings obtained by the routine diagnostic work-up in this cohort of patients. The primary hypothesis is that the detection rate of pathologic findings can be increased by undergoing enhanced diagnostic MRI work-up combined with prolonged Holter-monitoring. The primary outcome and all secondary outcomes are listed in Table 2, and a flowchart of study specific data acquisition is provided in Table 3 .

\section{Statistical analysis}

The sample size calculation is based on the aforementioned primary hypothesis (Table 2), that an enhanced diagnostic work-up consisting of cerebral and cardiac MRI (including contrast-enhanced angiography of the aortic arch and the carotid arteries) combined with a prolonged ECG-Monitoring (of up to 10 days duration) leads to a reduction in the number of strokes classified as cryptogenic from 30 to $25 \%$ when compared to the routine diagnostic work-up at the Stroke Unit. A sample size of 396 patients which receive both the enhanced as well as the routine diagnostic work-up would provide a power of $79 \%$ to detect a difference in the number of strokes classified as cryptogenic (from $30 \%$ after routine diagnostic work-up to $25 \%$ after enhanced diagnostic work-up, and with assumed $13 \%$ discordant pairs: $9 \%$ diagnosed as cryptogenic stroke by routine work-up but not according to enhanced diagnostic work-up, and $4 \%$

Table 2 Primary outcome and secondary outcomes of the HEBRAS study

\footnotetext{
Primary outcome

- Detection rate of pathologic findings relevant to stroke etiology in patients with acute ischemic stroke obtained by enhanced diagnostic MRI work-up combined with prolonged Holter-monitoring in comparison to findings obtained by routine diagnostic work-up.

Secondary outcomes

- To assess the benefit of prolonged continuous ECG-monitoring in AIS patients to detect paroxysmal AF.

- To determine the proportion of patients with first detected paroxysmal AF by prolonged Holter-monitoring (for up to 5 days) after hospital discharge.

- To identify the impact of stroke localization on autonomic changes (as indicated by elevated urinary norepinephrine levels and measures of HRV) or cardiac dysfunction (as indicated by troponin $T$ serum levels).

- To identify the impact of impaired HRV on recurrent vascular events and clinical outcome after AIS at 3 or 12 months after the index stroke, respectively.

- To assess the predictive value of imaging and biomarkers for AF-detection in patients with acute ischemic stroke.
}

diagnosed as cryptogenic stroke by enhanced work-up but not according to routine diagnostic work-up). The hypothesis will be tested using the McNemar-Test (twosided, $\alpha=0.05$ ). An interim analysis (with a significance level of $\alpha 1=0.004$ ) will be performed when 264 patients $(67 \%)$ are enrolled in the study. If $\mathrm{p}$ is $\leq \alpha 1$ at the interim analysis, the study will be terminated. When the study will be continued, the primary endpoint will be tested with $\alpha 2=0.046$ (two- tailed); the overall significance level will be $5 \%$. Taking into account a drop-out rate of about $20 \%, 475$ patients will have to be enrolled in the study.

\section{Study schedule}

The first patient was enrolled in May 2014. We anticipate a recruitment period of 18 months, and a recruitment period of additional 12 months is intended to enroll 475 patients With regard to the planned followup duration, first patient in to last-patient out will be 4.5 years in total.

\section{Discussion}

The primary aim of this prospective observational study is to clarify whether an enhanced diagnostic MRI workup combined with prolonged Holter-monitoring leads to a significant increase in relevant pathologic findings compared to guideline-recommended routine diagnostic work-up in patients with acute ischemic stroke without known atrial fibrillation before enrolment. There is a vital need to assess potential improvements in stroke care, because the etiology of about $20-25 \%$ of all ischemic strokes remains unknown [4]. The high percentage of unknown stroke etiology may be explained by low rates of transesophageal echocardiography [1, 15] and insufficient duration of ECG monitoring [12]. Compared to transesophageal echocardiography, cardiac MRI and MR angiography of the aortic arc are less invasive and show growing potential for a prominent diagnostic role in acute ischemic stroke [6]. However, due to lack of prospective studies, there are no clear cut recommendations for their clinical use in this context so far. This study aims to evaluate the efficacy of MRI in detecting embolic sources of stroke and thereby elucidate its future relevance for clinical routine.

Besides feasibility and diagnostic value of enhanced MRI, HEBRAS will assess the ability of extended systematic analysis of ECG-recording to improve AF detection compared to the current clinical standard, as similarly done in currently ongoing trials such as the Impact of Standardized MONitoring for Detection of Atrial Fibrillation in Ischemic Stroke Study (MonDAFIS)

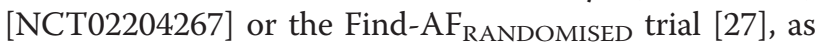
well as previous studies with shorter periods of monitoring $[28,29]$. Detection of paroxysmal AF in patients with acute ischemic stroke represents a major diagnostic 
Table 3 Study flowchart of the HEBRAS study

\begin{tabular}{|c|c|c|c|c|c|c|}
\hline & \multicolumn{4}{|c|}{ In-hospital stay } & \multicolumn{2}{|l|}{ Follow-up } \\
\hline & Admission & Day 1 & Day $\geq 2$ & Discharge & 3 months & 12 months \\
\hline Baseline data* & $x$ & & & & & \\
\hline Past medical history & $x$ & & & & $x$ & $x$ \\
\hline Heart rate at rest (routine) & $x$ & $x$ & Daily & $x$ & & \\
\hline Heart rhythm (routine) & $x$ & $x$ & Daily & $x$ & & \\
\hline 12-lead ECG & $x$ & & & & & \\
\hline 24-hour Holter & & & $(X)$ & & & \\
\hline Additional ECG monitoring ${ }^{a}$ & ongoing & & & ongoing $^{c}$ & & \\
\hline Respiratory rate & $x$ & $x$ & Daily & & & \\
\hline Clinical signs of infection & $x$ & & $x$ & $x$ & & \\
\hline Leukocytes [per mm³] & $x$ & & & & & \\
\hline Creatinine $[\mathrm{mg} / \mathrm{dl}]$ & $x$ & & & & & \\
\hline Potassium [mmol/l] & $x$ & & & & & \\
\hline Serum hs troponin I [ng/l] & $x$ & $x$ & & & & \\
\hline NT-proBNP [ng/l] & & $x$ & & & & \\
\hline Urine norepinephrine [nmol/l] & & $x$ & & & & \\
\hline Cardiac MRI & & $x$ & & & & \\
\hline MR angiography ${ }^{b}$ & & $x$ & & & & \\
\hline Brain MRI & & $x$ & & & & \\
\hline NIHSS & $x$ & & & $x$ & & \\
\hline Modified Rankin scale score & $x$ & & & $x$ & $x$ & $x$ \\
\hline Recurrent stroke, myocardial infarction or death of any cause & & & $x$ & $x$ & $x$ & \\
\hline
\end{tabular}

${ }^{a}$ see Methods section for more details

$\mathrm{b}_{\text {including the aortic arc and brain supplying arteries }}$

cextended for up to 5 days after hospital discharge/ transfer to a rehabilitation clinic

challenge nowadays, and AF remains undetected in a relevant proportion of stroke patients $[9,10]$. A faster assessment and better understanding of stroke etiology may improve secondary stroke prevention measures and thereby help to reduce the rate of stroke recurrence.

Another unique feature of the investigator-initiated HEBRAS study is the systematic assessment of strokeinduced autonomic dysfunction and cardiac damage by using a laboratory data. It has long been recognized that acute cerebrovascular events may coincide with or trigger cardiac dysfunction and elevation of cardiac biomarkers such as troponin. Several studies reported on elevation of cTn in patients with acute ischemic stroke [30-32]. However, although cTn is highly specific for myocardial injury, it does not reveal the underlying mechanism of injury. Elevations in cTn do not necessarily signify coronary myocardial ischemia. There are potential other causes of cTn elevations without underlying acute or chronic CAD [33]. One possible mechanism of acute cardiac injury in ischemic stroke, which pertains exclusively to patients with acute disease of the central nervous system, is neurogenic myocardial damage. Neurogenic myocardial damage may be caused by impairments within the central autonomic network. A crucial player of the central autonomic network would be the insula. Within a previous work, we have shown a positive association between stroke affecting the insula and troponin elevation [31]. Therefore, HEBRAS will elucidate the relationship between stroke location and troponin elevation with measures of voxel based lesion mapping.

In addition, natriuretic peptides (e.g., BNP) have been shown to be independently associated with atrial fibrillation in stroke cohorts [34-36], but have not been evaluated in large prospective trials thus far.

\section{Conclusion}

In summary, the HEBRAS study aims to clarify whether recent technical advantages can improve etiologic work up after acute ischemic stroke. This study has the potential to set essential modifications of current diagnostic standards after acute ischemic stroke, aiming at improving secondary stroke prevention. Moreover, the HEBRAS study will hopefully elucidate mechanisms of strokeinduced autonomic dysfunction and cardiac damage. 


\section{Abbreviations}

AF: Atrial fibrillation; bpm: Beats per minute; ECG: Electrocardiogramm; ESUS: Embolic Stroke of undetermined Source; FOV: Field of view; HEBRAS: HEart and BRain interfaces in Acute ischemic Stroke; HRV: Heart rate variability; HRV-TI: HRV-Triangular Index; LF: Low frequency; HF: High frequency; MRI: Magnetic resonance imaging; NASCET: North American symptomatic carotid endarterectomy trial; NYHA: New York heart association; s: Seconds; rMSSD: Root mean square of successive differences; SDNN: Standard deviation of beat-to-beat intervals; TE: Echo time; TEE: Transesophageal echocardiography; TOAST: Trial of ORG 10172 in acute stroke treatment; TR: Repetition time; TTE: Transthoracal echocardiography.

\section{Competing interest}

None of the authors have any competing interests.

\section{Authors' contributions}

All authors have 1) made substantial contributions to conception and design of the study, 2) have been involved in drafting the manuscript or revising it critically for important intellectual content; 3 ) have given final approval of the version to be published; and 4) agree to be accountable for all aspects of the work in ensuring that questions related to the accuracy or integrity of any part of the work are appropriately investigated and resolved.

\section{Acknowledgements}

We thank Kristin Simon (CSB) and Dr. Juliane Herm (CSB) for their support of study conduct. Furthermore, we thank Julia Herde (CSB) for critically reviewing the manuscript.

\section{Financial disclosures}

KGH reports lecture fees and a study grants by Bayer Healthcare, lecture fees and a study grant by Sanofi as well as lecture fees from Pfizer and Bristol-Myers Squibb.

ME reports lecture fees and study grants by Bayer, Boehringer Ingelheim, Bristol-Myers-Squibb, Ever, Glaxo Smith Kline, MSD, Novartis and Pfizer. TK and UG have no financial disclosures to report.

$\mathrm{CHN}$ reports lecture fees from Pfizer, Boehringer Ingelheim, Bristol-Myers Squibb and Bayer Pharma.

JBF reports the following consultancies and/or payments for lectures: BoehringerIngelheim, Lundbeck, BioClinica and Parexel.

\section{Grants}

This study is supported by the BMBF (German Ministry of Education and Research) [grant G.2.17, Center for Stroke Research Berlin], by the DZHK (German Center for Cardiovascular Research) [grant B14-035_SE] and by the GETEMED AG, Teltow, Germany.

\section{Author details}

'Center for Stroke Research Berlin, Charité - Universitätsmedizin Berlin, Berlin, Germany. 'Department of Neurology, Campus Benjamin Franklin, Charité Universitätsmedizin Berlin, Hindenburgdamm 30, 12200 Berlin, Germany. ${ }^{3}$ Excellence Cluster NeuroCure, Charité - Universitätsmedizin Berlin, Berlin, Germany. ${ }^{4}$ German Center for Neurodegenerative Diseases, Berlin, Germany. ${ }^{5}$ German Center for Cardiovascular Diseases, Partner Site Berlin, Berlin, Germany. ${ }^{6}$ Berlin Institute of Health, Berlin, Germany. ${ }^{7}$ Department for Biostatistics and Clinical Epidemiology, Charité - Universitätsmedizin Berlin, Berlin, Germany.

\section{Received: 21 August 2015 Accepted: 3 October 2015}

Published online: 22 October 2015

\section{References}

1. Heidrich J, Heuschmann PU, Kolominsky-Rabas P, Rudd AG, Wolfe CD, European BIISOSCG. Variations in the use of diagnostic procedures after acute stroke in Europe: results from the BIOMED II study of stroke care. Eur J Neurol. 2007;14(3):255-61.

2. Herm J, Konieczny M, Jungehulsing GJ, Endres M, Villringer A, Malzahn U, et al. Should transesophageal echocardiography be performed in acute stroke patients with atrial fibrillation? J Clin Neurosci. 2013;20(4):554-9.

3. Rizos T, Rasch C, Jenetzky E, Hametner C, Kathoefer S, Reinhardt R, et al. Detection of paroxysmal atrial fibrillation in acute stroke patients. Cerebrovasc Dis. 2010;30(4):410-7.
4. Hart RG, Diener HC, Coutts SB, Easton JD, Granger CB, O'Donnell MJ, et al. Embolic strokes of undetermined source: the case for a new clinical construct. Lancet Neurol. 2014;13(4):429-38.

5. Barkhausen J, Hunold P, Eggebrecht H, Schuler WO, Sabin GV, Erbel R, et al. Detection and characterization of intracardiac thrombi on MR imaging. AJR Am J Roentgenol. 2002;179(6):1539-44.

6. Pagan RJ, Parikh PP, Mergo PJ, Gerber TC, Mankad R, Freeman WD, et al. Emerging role of cardiovascular CT and MRI in the evaluation of stroke. AJR Am J Roentgenol. 2015;204(2):269-80.

7. Harloff A, Dudler P, Frydrychowicz A, Strecker C, Stroh AL, Geibel A, et al. Reliability of aortic MRI at 3 Tesla in patients with acute cryptogenic stroke. J Neurol Neurosurg Psychiatry. 2008;79(5):540-6.

8. Nederkoorn PJ, van der Graaf Y, Hunink MG. Duplex ultrasound and magnetic resonance angiography compared with digital subtraction angiography in carotid artery stenosis: a systematic review. Stroke. 2003;34(5):1324-32.

9. Gladstone DJ, Spring M, Dorian P, Panzov V, Thorpe KE, Hall J, et al. Atrial fibrillation in patients with cryptogenic stroke. N Engl J Med. 2014;370(26):2467-77.

10. Sanna T, Diener HC, Passman RS, Di Lazzaro V, Bernstein RA, Morillo CA, et al. Cryptogenic stroke and underlying atrial fibrillation. N Engl J Med. 2014;370(26):2478-86.

11. Glotzer TVZP. Silent atrial fibrillation as a stroke risk factor and anticoagulation indication. Can J Cardiol. 2013;7(29):S14-23.

12. Rizos T, Quilitzsch A, Busse O, Haeusler KG, Endres M, Heuschmann P, et al. Diagnostic work-up for detection of paroxysmal atrial fibrillation after acute ischemic stroke: cross-sectional survey on german stroke units. Stroke. 2015;46(6):1693-5.

13. Jauch EC, Saver JL, Adams Jr HP, Bruno A, Connors JJ, Demaerschalk BM, et al. Guidelines for the early management of patients with acute ischemic stroke: a guideline for healthcare professionals from the American Heart Association/American Stroke Association. Stroke. 2013:44(3):870-947.

14. January CT, Wann LS, Alpert JS, Calkins H, Cigarroa JE, Cleveland Jr JC, et al. 2014 AHA/ACC/HRS guideline for the management of patients with atrial fibrillation: executive summary: a report of the American College of Cardiology/American Heart Association Task Force on practice guidelines and the Heart Rhythm Society. Circulation. 2014;130(23):2071-104.

15. Dittrich R, Schmidt WP, Heidrich J, Bucker-Nott HJ, Ringelstein EB, Heuschmann PU, et al. Differences in the extent of diagnostic procedures after acute stroke in patients treated in departments of neurology, internal medicine and geriatric medicine. Fortschr Neurol Psychiatr. 2005;73(2):68-73.

16. Scheitz JF, Erdur H, Haeusler KG, Audebert HJ, Roser M, Laufs U, et al. Insular cortex lesions, cardiac troponin, and detection of previously unknown atrial fibrillation in acute ischemic stroke: insights from the troponin elevation in acute ischemic stroke study. Stroke. 2015;46(5):1196-201.

17. Scheitz JF, Mochmann HC, Erdur H, Tutuncu S, Haeusler KG, Grittner U, et al. Prognostic relevance of cardiac troponin $\mathrm{T}$ levels and their dynamic changes measured with a high-sensitivity assay in acute ischaemic stroke: analyses from the TRELAS cohort. Int J Cardiol. 2014;177(3):886-93.

18. Adams Jr HP, Bendixen BH, Kappelle LJ, Biller J, Love BB, Gordon DL, et al. Classification of subtype of acute ischemic stroke. Definitions for use in a multicenter clinical trial. TOAST. Trial of Org 10172 in Acute Stroke Treatment. Stroke. 1993;24(1):35-41.

19. von Reutern GM, Goertler MW, Bornstein NM, Del Sette M, Evans DH, Hetzel A, et al. Grading carotid stenosis using ultrasonic methods. Stroke. 2012:43(3):916-21.

20. Bates E, Wilson SM, Saygin AP, Dick F, Sereno MI, Knight RT, et al. Voxel-based lesion-symptom mapping. Nat Neurosci. 2003;6(5):448-50.

21. Rorden $\mathrm{C}$, Karnath $\mathrm{HO}$, Bonilha L. Improving lesion-symptom mapping. J Cogn Neurosci. 2007;19(7):1081-8.

22. Camm AJ, Lip GY, De Caterina R, Savelieva I, Atar D, Hohnloser SH, et al. 2012 focused update of the ESC Guidelines for the management of atrial fibrillation: an update of the 2010 ESC Guidelines for the management of atrial fibrillation. Developed with the special contribution of the European Heart Rhythm Association. Eur Heart J. 2012;33(21):2719-47.

23. Wallmann D, Tuller D, Wustmann K, Meier P, Isenegger J, Arnold M, et al. Frequent atrial premature beats predict paroxysmal atrial fibrillation in stroke patients: an opportunity for a new diagnostic strategy. Stroke. 2007;38(8):2292-4.

24. Heart rate variability: standards of measurement, physiological interpretation and clinical use. Task force of the european society of cardiology and the North American society of pacing and electrophysiology. Circulation. 1996, 93(5):1043-65. 
25. Brott T, Adams Jr HP, Olinger CP, Marler JR, Barsan WG, Biller J, et al. Measurements of acute cerebral infarction: a clinical examination scale. Stroke. 1989;20(7):864-70

26. van Swieten JC, Koudstaal PJ, Visser MC, Schouten HJ, van Gijn J. Interobserver agreement for the assessment of handicap in stroke patients. Stroke. 1988;19(5):604-7.

27. Weber-Kruger M, Gelbrich G, Stahrenberg R, Liman J, Kermer P, Hamann GF, et al. Finding atrial fibrillation in stroke patients: Randomized evaluation of enhanced and prolonged Holter monitoring-Find-AF(RANDOMISED) -rationale and design. Am Heart J. 2014;168(4):438-45. e431.

28. Grond M, Jauss M, Hamann G, Stark E, Veltkamp R, Nabavi D, et al. Improved detection of silent atrial fibrillation using 72-hour Holter ECG in patients with ischemic stroke: a prospective multicenter cohort study. Stroke. 2013;44(12):3357-64

29. Rizos T, Guntner J, Jenetzky E, Marquardt L, Reichardt C, Becker R, et al. Continuous stroke unit electrocardiographic monitoring versus 24-hour Holter electrocardiography for detection of paroxysmal atrial fibrillation after stroke. Stroke. 2012;43(10):2689-94.

30. Faiz KW, Thommessen B, Einvik G, Brekke PH, Omland T, Ronning OM. Determinants of high sensitivity cardiac troponin T elevation in acute ischemic stroke. BMC Neurol. 2014;14:96.

31. Scheitz JF, Endres M, Mochmann HC, Audebert HJ, Nolte CH. Frequency, determinants and outcome of elevated troponin in acute ischemic stroke patients. Int J Cardiol. 2012;157(2):239-42.

32. Feher G, Tibold A, Koltai K, Szapary L. The clinical importance of troponin elevation in ischaemic cerebrovascular events: a clinical review. J Cardiol Ther. 2014;7(1):141-9.

33. Scheitz JF, Nolte $\mathrm{CH}$, Laufs U, Endres M. Application and interpretation of high-sensitivity cardiac troponin assays in patients with acute ischemic stroke. Stroke. 2015;46(4):1132-40.

34. Shibazaki K, Kimura K, Fujii S, Sakai K, Iguchi Y. Brain natriuretic peptide levels as a predictor for new atrial fibrillation during hospitalization in patients with acute ischemic stroke. Am J Cardiol. 2012;109(9):1303-7.

35. Suissa L, Bresch S, Lachaud S, Mahagne MH. Brain natriuretic peptide: a relevant marker to rule out delayed atrial fibrillation in stroke patient. J Stroke Cerebrovasc Dis. 2013;22(7):e103-10

36. Wachter R, Lahno R, Haase B, Weber-Kruger M, Seegers J, Edelmann F, et al. Natriuretic peptides for the detection of paroxysmal atrial fibrillation in patients with cerebral ischemia-the Find-AF study. PLoS One. 2012;7(4):e34351.

37. Association TCCotNYH. Functional capacity and objective assessment. In: Dolgin M, editor. Nomenclature and criteria for diagnosis of diseases of the heart and great vessels. 9th ed. Boston, MA: Little, Brown and Company; 1994. p. 253-5.

\section{Submit your next manuscript to BioMed Central and take full advantage of:}

- Convenient online submission

- Thorough peer review

- No space constraints or color figure charges

- Immediate publication on acceptance

- Inclusion in PubMed, CAS, Scopus and Google Scholar

- Research which is freely available for redistribution 